\title{
Balance de resultados en la aplicación de metodologías activas de aprendizaje en la asignatura de Máquinas Hidráulicas (Grado de Ingeniería en Tecnologías Industriales)
}

\author{
Rosa M. Llácer-Iglesias, Estíbaliz Fernández de Velasco Sanz y F. Javier Martínez- \\ Solano \\ Departamento de Ingeniería Hidráulica y Medio Ambiente, Universitat Politècnica de València. \\ rollaig@hma.upv.es; esferde@dihma.upv.es; jmsolano@upv.es.
}

\begin{abstract}
After three years since the flipped teaching methodology started to be applied, the results might be considered as quite good. The progressive modification of resources and methods in order to reinforce aspects that are considered key, such as the students' awareness of the change in methodology and the obtaining of specific feedback on reverse teaching, have contributed significantly to the improvement of results in its application. In the same way, the introduction to additional resources, such as gamification, have also had a positive impact.The marks obtained by the students together with the successive modifications and continuous improvements introduced since then allow to draw conclusions in a more general way. These conclusions are likely to be extended to other subjects in a wide range of fields.
\end{abstract}

Keywords: laboratory class; flipped teaching; gamification; self-learning

\begin{abstract}
Resumen
Transcurridos tres años desde la aplicación por primera vez de metodologías activas de aprendizaje en las prácticas de la asignatura Máquinas Hidráulicas, el balance global se considera muy positivo. La modificación progresiva de materiales y métodos con el fin de reforzar aspectos que se consideran clave, tales como la toma de conciencia por parte del alumnado del cambio de metodología y la obtención de retroalimentación específica sobre la docencia inversa han contribuido notablemente a la mejora de resultados en su aplicación. La introducción además de recursos adicionales, como la gamificación también han tenido una repercusión positiva. Los resultados obtenidos y las sucesivas modificaciones y mejoras introducidas desde entonces, permiten extraer conclusiones de una forma más general y por tanto, probablemente extensibles a otras asignaturas, en muy diferentes áreas de conocimiento.
\end{abstract}

Palabras clave: prácticas de laboratorio; docencia inversa; gamificación; aprendizaje autónomo 


\section{Introducción}

Durante el curso 2015-2016, los autores de este trabajo empezaron a implantar la metodología de Docencia Inversa en la asignatura de Máquinas Hidráulicas, obligatoria del Grado en Ingeniería de Tecnologías Industriales, correspondiente al primer semestre del último curso ( $\left.4^{\circ} \mathrm{A}\right)$ (Llácer-Iglesias \& Martínez-Solano, 2016). Esta asignatura tiene un elevado número de alumnos matriculados (280 en el curso 2017-2018, con una media cercana a los 290 en el balance de los últimos 5 años) repartidos en cinco grupos de teoría. Estos grupos de teoría se dividen a su vez en tres grupos de prácticas dando como resultado 15 grupos de prácticas con un número medio de alumnos de 19 alumnos por grupo, aunque por los distintos horarios ofrecidos, la distribución entre ellos no es demasiado homogénea, dando lugar tanto a grupos reducidos de 8-10 alumnos, como a grupos numerosos con hasta 25 asistentes. La asignatura consta de 4,5 créditos ECTS (2 ECTS de lección magistral, 1,6 de prácticas en el aula, 0,3 de prácticas en laboratorio y 0,6 de prácticas informáticas).

La asignatura se engloba dentro de un Grado diseñado en el marco del Espacio Europeo de Educación Superior (EEES), también conocido como Plan Bolonia. Desde el principio, el interés de la Escuela Técnica Superior de Ingenieros Industriales ha buscado la inclusión de técnica innovadoras de enseñanza aprendizaje. Esta misma inquietud fue asimilada por el equipo docente, compuesto en la actualidad por 8 profesores. Y así, durante el curso 20152016 se comenzó a introducir la metodología de la docencia inversa en la asignatura (Llácer-Iglesias \& Martínez-Solano, 2016).

En su planteamiento, el primer desafío con el que se encontró el equipo docente fue el elevado número de alumnos matriculados y de grupos, que claramente dificultaban las labores de coordinación y seguimiento. Ante esta perspectiva, la inversión de recursos para abordar la implantación de esta metodología en la totalidad de la asignatura se presentaba como un proyecto excesivamente ambicioso e incluso arriesgado. Sin embargo, el interés por introducir este tipo de innovaciones hizo que finalmente se plantease llevarlo a cabo, a modo de experiencia piloto, en una pequeña parte de la asignatura. Por ser especialmente significativa se eligió para ello las prácticas de laboratorio. Puesto que además en el curso anterior se había reducido el número de créditos presenciales para esta práctica, se consideró que la utilización de la docencia inversa para su preparación previa podría permitir un mayor aprovechamiento del tiempo en el laboratorio.

Hasta el curso anterior al de inicio de la experiencia, estas prácticas constaban de 2 sesiones de 2,5 horas de duración cada una, en las que se llevaban a cabo un total de 2 ó 3 ensayos. En función del número de alumnos asistentes, como se ha comentado muy variable, y de su predisposición, el ritmo de las mismas podía ser muy diferente. Con este planteamiento, en años anteriores se venían observando además en muchos casos esperas y tiempos muertos, que generalmente no eran aprovechados por los alumnos. A ello se añadía que aunque habitualmente el material de prácticas se encontraba disponible con anterioridad a su realización, su preparación no era una práctica habitual entre los estudiantes, a pesar de la importancia que conlleva. Por tanto al inicio de las sesiones resultaba necesario consumir una cantidad importante de tiempo para exponer todos los contenidos y los procedimientos de operación en cada ensayo, y aún así, se seguían produciendo tiempos inactivos o no

(cc) EY-NC-ND 2018, Universitat Politècnica de València

Congreso IN-RED (2018) 
válidos durante su realización, por no haber comprendido el alumno en esa explicación la operativa a seguir.

Por ello, se decidió que una buena forma de aprovechar mejor el tiempo de permanencia en el laboratorio pasaba por asegurarse de que los alumnos entraban con los fundamentos básicos ya asimilados. Ahí surge la posibilidad de utilizar los conceptos típicos de la docencia inversa. Sams \& Bergmann (2013) propusieron en el año 2007 un sistema mediante el cual los alumnos que tenían y que por cualquier razón no podían asistir a una clase tuvieran la posibilidad de recuperarla mediante el visionado de grabaciones de la misma. A la vista de los resultados, extendieron la experiencia al resto de los alumnos, permitiendo que las partes más básicas del temario fuese preparada por estos también en ese caso e incidiendo posteriormente en clase en aspectos más complejos o aplicados.

Por todo ello, se consideró que las prácticas de laboratorio podrían ser la parte de la asignatura que podía encontrar mayores beneficios en la aplicación piloto de esta nueva metodología docente, ya que, además de fomentar activamente el autoaprendizaje, se intentaba también que el tiempo que el alumno permanecía en el laboratorio fuera aprovechado al máximo. De este modo, el desarrollo de los ensayos hace ahora 3 años se concentró en una única sesión de 3 horas y el primer año se elaboró nuevo material que facilitase la preparación previa, el apoyo durante su realización y análisis de resultados posterior.

Así, en el curso 2015-2016 se puso en práctica una experiencia piloto de aplicación de técnicas típicas de la docencia inversa a las prácticas de laboratorio de la asignatura de Máquinas Hidráulicas de $4^{\circ}$ curso del Grado de Ingenerio en Tecnolocías Industriales (GITI).

La práctica de laboratorio consiste en la realización de ensayos en 3 montajes de circuitos hidráulicos sencillos para caracterización de las bombas que los componen y aplicación de algunos conceptos básicos de su funcionamiento y operación. Estos ensayos son llevados a cabo en equipo, formando como máximo 3 (uno para cada montaje) entre todos los asistentes a la sesión. El número de miembros en cada equipo puede variar entre 4 y 9 en función del total de alumnos en el grupo. Los alumnos van realizando de forma rotativa los ensayos, de modo que cada equipo empieza en un montaje distinto, y al finalizar la sesión todos ellos han llevado a cabo los 3 ensayos.

En todos los casos, el objetivo principal es familiarizar al alumno con los métodos de ensayo de bombas para la obtención de sus curvas características H-Q, P-Q y १-Q, así como con la instrumentación a emplear. Existen también objetivos adicionales relacionados con otros conceptos importantes expuestos en el temario. En concreto actualmente los tres bancos de trabajo consisten en:

- Banco 1: Ensayo de bomba centrífuga en aspiración. Caracterización de la bomba.

- Banco 2: Ensayo de bomba sumergida con variador de frecuencia. Caracterización de la bomba a diferentes frecuencias y aplicación de la teoría de semejanza. 
- Banco 3: Ensayo de dos bombas centrífugas en carga, con opción de funcionamiento simultáneo en asociación en serie o en paralelo. Caracterización de una bomba individual y caracterización de ambas asociaciones.

La aplicación de la docencia inversa ese primer año en las clases de prácticas de laboratorio de la asignatura funcionó bastante bien, consiguiéndose mejoras en las calificaciones no solo en la parte práctica sino también y de manera más significativa en los conceptos propios de las lecciones magistrales. Sin embargo, se observaron tres áreas de mejora que se han intentado aplicar desde el curso 2016-2017 (Llácer-Iglesias \& Martínez-Solano, 2016):

- Concienciación. Se detectó una falta de familiaridad de los alumnos con la nueva metodología. Se hacía por tanto necesario realizar un esfuerzo didáctico de la importancia del trabajo previo para garantizar el éxito de la misma.

- Retroalimentación. Más allá de las calificaciones, resultaba importante conocer la percepción que los alumnos tenían de la nueva metodología.

- Evaluación. Se planteó como forma de comprobar el trabajo previo la posibilidad de contrastar hasta qué punto se habían adquirido ciertos conceptos básicos.

Por último, también se detectó falta de familiaridad de los alumnos con determinados protocolos más propios del ámbito de la prevención de riesgos laborales que de la propia hidráulica. Esta percepción, sin embargo, no es nueva. González Rogado et al. (2014) también han detectado lagunas en este sentido. En la Universidad Politécnica de Valencia (UPV) existe un Servicio Integrado de Prevención y Salud Laboral que se encarga entre otras funciones de elaborar guías prácticas dirigidas tanto a alumnos como a personal de la Universidad. Estas guías son específicas de cada tipo de laboratorio o taller, distinguiendo entre riesgos químicos, eléctricos y mecánicos. Sin embargo, no hay un control real del seguimiento de estas guías por parte del alumnado. De hecho, en muchos casos el alumnado desconoce su existencia. Por ello, se decidió que, puesto que no forma parte del temario de la asignatura pero sí constituyen una parte fundamental de los conocimientos que deben adquirirse antes de empezar a trabajar en el laboratorio, era una buena oportunidad de aplicar la docencia inversa.

Además, si bien no está incluida entre las 13 competencias transversales que la UPV evalúa, la competencia de prevención de riesgos en los laboratorios constituye una competencia que debería ser evaluada en algún punto de la titulación. En esta línea se desarrolla también el trabajo desarrollado por González Rogado et al. (2014) en la Universidad de Salamanca. En dicho trabajo, estos autores realizan una evaluación de la competencia antes de entrar en el laboratorio.

\section{Objetivos}

El objetivo principal de la presente contribución es el de presentar, tras tres años de experiencia, el balance de resultados y conclusiones en la aplicación de la docencia inversa a las prácticas de laboratorio de la asignatura Máquinas Hidráulicas del $4^{\circ}$ curso del Grado en Ingeniería de Tecnologías Industriales, de modo que pueda animar a docentes de muy

(cc) EY-NC-ND 2018, Universitat Politècnica de València

Congreso IN-RED (2018) 
diversas áreas a introducir de forma gradual y sencilla aspectos basados en esta metodología, sin necesidad de recurrir a herramientas complejas o a tener que reestructurar por completo sus asignaturas. Esta posibilidad puede ser una opción especialmente interesante en el caso de asignaturas con un elevado número de alumnos y numerosos grupos como en el caso que nos ocupa.

Para conseguir dicho objetivo se han planteado los siguientes objetivos específicos:

- Evaluar la adecuación de las materiales y métodos utilizados.

- Analizar el impacto sobre los resultados de las distintas modificaciones y mejoras introducidas de forma sucesiva a lo largo de estos tres años, tanto mediante análisis de resultados académicos como con la información aportada por los alumnos a través de encuestas.

- Analizar los beneficios observados y las principales limitaciones encontradas, tanto de forma general, como específica a la aplicación de esta metodología a prácticas de laboratorio.

\section{Desarrollo de la innovación}

\subsection{Materiales desarrollados}

\subsubsection{Documentación escrita: instrucciones y procedimientos}

Si bien los métodos audiovisuales son los más frecuentemente utilizados cuando nos encontramos con experiencias relacionadas con la docencia inversa, ya el primer año se apostó por la utilización de materiales más sencillos, basados en documentos, procedimientos e instrucciones de operación escritas por varios motivos.

En primer lugar, por el firme convencimiento de los autores de que al menos parte de la formación del alumno, debería continuar basándose en la lectura, como forma más activa de autoaprendizaje. El riesgo de basarse únicamente en materiales audiovisuales y presentaciones, que en cierto modo pudiesen alejar al alumno de ingeniería del hábito fundamental de lectura durante su formación académica, era uno de los principales temores al inicio de la experiencia. Entre el profesorado de la asignatura se encuentran profesionales del mundo de la industria que recalcaban la importancia de incluir actividades que continuaran fomentando la comprensión lectora como una actividad más del proceso de formación y adquisición de competencias por parte de los estudiantes. Igualmente importante se consideró en el ámbito académico, habiendo observado a lo largo de los años casos frecuentes de fallos importantes en actos de evaluación, por el mero hecho de no haber realizado una correcta lectura de las cuestiones planteadas. Teniendo en cuenta que en la actualidad en todos los ámbitos existe una mayor tendencia a la utilización de métodos audiovisuales, se consideró conveniente adoptar documentación escrita para esta asignatura, de modo que se ampliara el espectro de herramientas y métodos a utilizar en el autoaprendizaje continuo que el desarrollo de su carrera profesional va a requerir una vez se incorporen al mundo profesional.

En segundo lugar, porque la experiencia desde el primer año ha mostrado que para el caso concreto de prácticas de laboratorio, y en particular para las de esta asignatura, que cuenta 
con un elevado número de alumnos, este planteamiento podría optimizar los resultados, en cuanto a aprovechamiento y rendimiento académico obtenido de las horas presenciales en el laboratorio por parte del alumno. Visto que los alumnos deben llevar a cabo los ensayos por sí mismos, las numerosas variables a tener en cuenta y que la sesión de prácticas tiene un tiempo limitado, se observó que resultaba inevitable tener que realizar al inicio por parte de los profesores una breve introducción y una revisión rápida de los procedimientos a llevar a cabo en los distintos bancos de ensayo, con el fin de transmitirles una cierta seguridad en lo que van a trabajar. En caso de basar la preparación previa en la visualización de grabaciones multimedia realizando esas mismas explicaciones, básicamente consistiría en una repetición, lo que se consideró que no resultaría tan enriquecedor. Al mismo tiempo, dicha introducción podría transmitirles mayor confianza y contribuir a la interacción alumnos/profesor a lo largo de toda la sesión.

De este modo, finalmente se decidió que todo el material de las prácticas fuera documentación escrita, elaborada de tal forma que sirviese de apoyo al alumno, tanto para su preparación (antes de la sesión), como de guía durante la realización de los ensayos y en el análisis de resultados y conclusiones posterior a la práctica.

El material que sirve de soporte a las prácticas debe permitir al alumno familiarizarse con el mismo antes de haber entrado en el laboratorio. Por ello, debe cumplir una serie de requisitos:

- Debe estar muy estructurado, manteniendo la secuencia lógica de contenidos. Debe ser claro y conciso, pero completo a la vez. Es decir, no debe contener excesiva información, que no sea necesaria para la práctica, pero sí toda la suficiente para su correcto desarrollo.

- Debe ser fiel al objeto, equipamiento y procedimiento a seguir. Dicho de otra forma, debe describir exactamente los equipos e instrumentación que se van a utilizar y los ensayos a realizar. Es fundamental que el material fuese totalmente adaptado a lo que los alumnos encontrarán en el laboratorio y a las actividades que realizarán durante la práctica. Esto implica una continua actualización del material para responder a las necesarias variaciones en los montajes de prácticas de laboratorio.

Puesto que a lo largo de estos tres años se han producido modificaciones importantes en los ensayos a realizar (cambios de equipos y metodología de obtención de datos), lógicamente este material se ha ido actualizando año tras año, manteniendo la misma esencia, pero con los cambios necesarios para reflejar exactamente lo que el alumno iba a encontrarse en el laboratorio y debía hacer durante la práctica.

Inicialmente el primer año la documentación se estructuró en 3 partes. La Parte I, Normas de seguridad y prevención de riesgos en el ambiente de trabajo, formaba parte del protocolo de acceso al laboratorio. Si bien no queda reflejado en ningún sitio, desde la asignatura se propuso desde el principio la necesidad de formar a los alumnos en la prevención de riesgos laborales. Por ello, para acceder al laboratorio era necesario en primer lugar entregar cumplimentado previamente un test, Anexo a dicha parte, en el que se les planteaban

(cc) EY-NC-ND 2018, Universitat Politècnica de València

Congreso IN-RED (2018) 
cuestiones básicas relacionadas con la naturaleza y origen de los riesgos en el mismo. La Parte II describía los protocolos y procedimientos necesarios para la realización de los ensayos, y también llevaba un Anexo con cuestiones a entregar cumplimentado al inicio de la sesión. Por último, la Parte III incluía las tablas con los datos que deben ser recogidos en el laboratorio, así como los cálculos y resultados que debían entregarse al finalizar la sesión para completar la evaluación. Todo el material estaba disponible en la plataforma PoliformaT con anterioridad suficiente a la sesión.

No obstante, con esta distribución y con la reestructuración de la práctica de laboratorio en una única sesión, se observaron resultados muy dispares entre los 15 grupos, dependiendo no sólo del grado de preparación de los miembros de cada equipo de trabajo, sino también en parte del número de asistentes totales a la sesión. En grupos en los que el número de alumnos total superaba la veintena, las posibles incidencias o lentos desarrollos de un equipo tenía una influencia importante en los otros dos. Además de esta circunstancia, se consideró también que el aprovechamiento, tanto de las horas de laboratorio, como del aprendizaje de la práctica en su conjunto, podría optimizarse y repartirse de forma más homogénea entre todos los alumnos, incluyendo un análisis de resultados más detallado posterior a la práctica. De este modo una de las modificaciones del segundo año incluyó un cambio en la distribución de la documentación y en base a ello, de la evaluación.

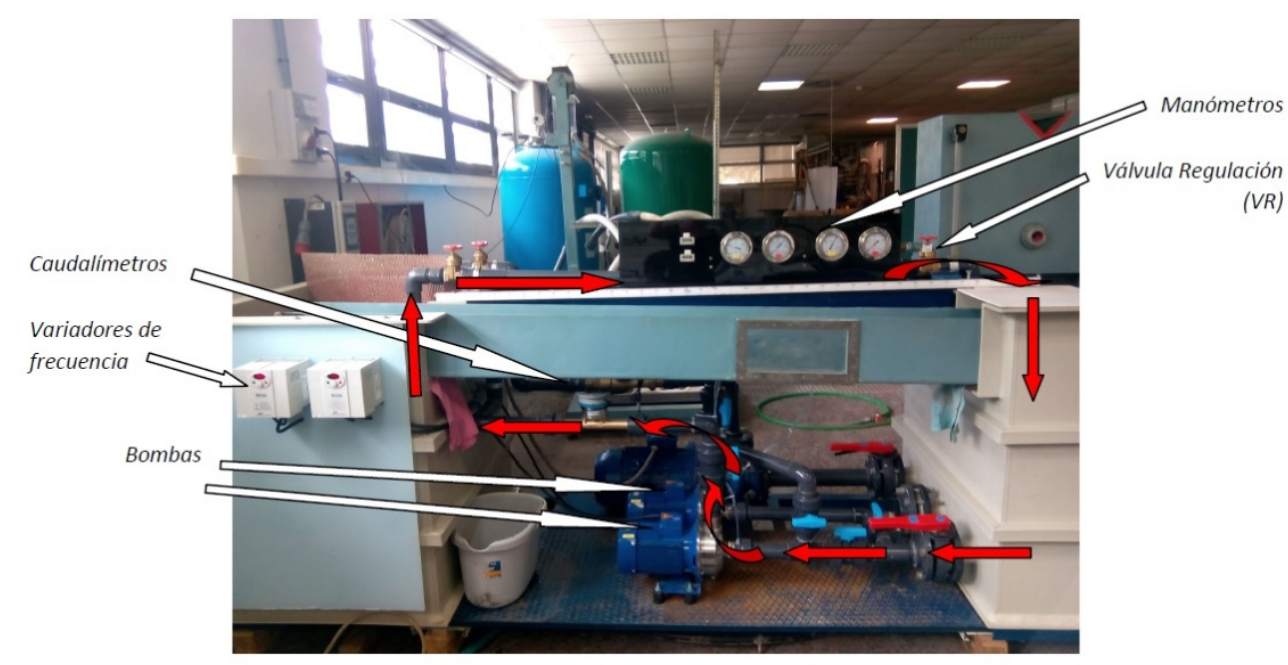

Figura 1. Ejemplo de fotografía incluida en el documento Parte II, en la descripción del banco de trabajo (Ensayo $n^{\circ}$ 3: Asociación en serie-paralelo).

Así, la documentación actualmente se estructura del siguiente modo:

- Parte I: Riesgos y normas de seguridad en el laboratorio.

- Parte II: Documento explicativo de los ensayos, incluyendo para cada uno de ellos una introducción teórica, descripción del circuito hidráulico y equipos (ver Figura 1) y procedimiento operativo para llevar a cabo el ensayo.

- Parte III: Hojas de toma de datos experimentales y cálculos preliminares. 
- Parte IV: Cálculos y análisis de resultados.

Y las entregas para evaluación se han distribuido del siguiente modo:

- Al inicio de la práctica (como trabajo previo): Se ha mantenido la entrega de forma individual y cumplimentada a mano de los Anexos correspondientes a las partes I y II.

- Al finalizar la sesión (a modo de control como trabajo durante la misma): Por cada equipo de trabajo, la parte III, ahora únicamente con datos experimentales y un par de cálculos sencillos.

- A las 5-6 semanas de finalizar las prácticas (como trabajo posterior): También por cada equipo de trabajo, entrega de archivo con cálculos y análisis más detallado de resultados.

\subsubsection{Encuestas}

Otra de las modificaciones introducidas el segundo año, tiene que ver con el aumento de la retroalimentación por parte del alumnado, sobre aspectos específicos de la metodología de docencia inversa, como una de las áreas de mejora identificadas inicialmente (LlácerIglesias \& Martínez-Solano, 2016).

Para ello, durante el curso 2016-2017 se elaboró una encuesta interna, basada en la estructura de las utilizadas de forma general en la UPV para la evaluación del profesorado y con los mismos baremos de puntuación, pero añadiendo un par de preguntas específicas sobre la docencia inversa (ver figura a continuación).

Docencia:

Programación: Antes de la práctica se proporciona información clara sobre la misma (objetivos, programa, metodología y criterios de evaluación).

Contenido: Las actividades realizadas en la práctica son de gran ayuda para el aprendizaje de la asignatura.

Profesorado: La profesora contribuye a crear un buen clima de trabajo y anima a los alumnos a participar en las clases.

Profesorado: Teniendo en cuenta las limitaciones, pienso que la profesora que imparte esta práctica debe considerarse una buena profesora.

\section{Metodología Docente (Docencia Inversa):}

Material: El material docente habilitado en PoliformaT para la práctica es coherente con los contenidos del programa, representa una importante ayuda para su aprendizaje y para alcanzar los objetivos.

Metodología: Teniendo en cuenta que la docencia inversa implica un trabajo previo a la práctica con ayuda del material disponible, y viendo lo que he aprendido, considero que es una metodología útil para el aprendizaje y adecuada para estas prácticas de laboratorio.
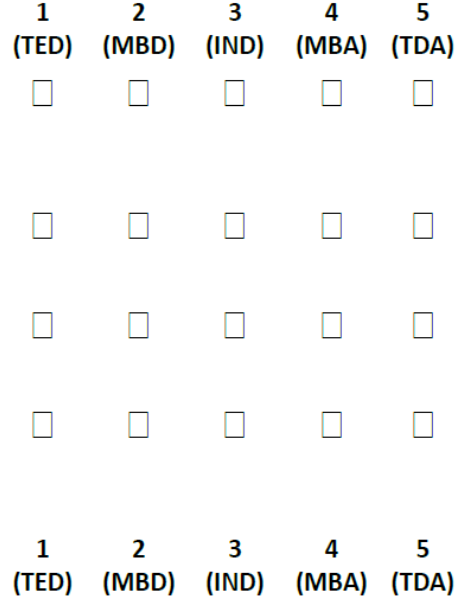

Figura 2. Preguntas sobre docencia inversa en encuesta interna realizada el curso 2016-2017.

(c)) EY-NC-ND 2018, Universitat Politècnica de València

Congreso IN-RED (2018) 
Para intentar ampliar dicha información, con detalles más específicos sobre la docencia inversa, en el tercer año (curso 2017-2018) se optó por no realizar encuesta interna, sino llevar a cabo la encuesta prevista por el Proyecto Docencia Inversa.

\subsection{Métodos utilizados}

\subsubsection{Labor de concienciación}

Una importante lección aprendida el primer año de la experiencia, fue la identificación de la necesidad de concienciar a los alumnos desde el inicio de la asignatura del propio concepto de docencia inversa, ya que se observó que muchos de ellos ni siquiera eran conscientes de que se va a utilizar dicha metodología en la asignatura en la que se habían matriculado (Llácer-Iglesias \& Martínez-Solano, 2016). Para abordar esta cuestión, en el segundo curso que se empleó docencia inversa, se reforzó la información a los alumnos mediante las siguientes acciones:

- A modo de introducción, el primer día de la asignatura, el profesor de teoría correspondiente, recalcó desde el inicio, de que en las prácticas de laboratorio se emplearía esta metodología.

- Transcurridas unas semanas, pero con 10-15 días de antelación a la realización de las prácticas, durante una de las clases teóricas, los profesores de laboratorio realizaron una presentación específica de las prácticas, explicando con detalle objeto, contenido, materiales, metodología y métodos de evaluación, y haciendo especial hincapié en la importancia de preparar con antelación la sesión conforme a las pautas expuestas. Con el fin de aumentar la motivación a la preparación de la sesión, se planteó la posibilidad de que se realizasen preguntas, aleatorias dado el elevado número de alumnos, sobre el procedimiento que tendrían que seguir, que formarían parte de la evaluación.

Aunque las presentaciones realizadas el segundo año con anterioridad a la práctica ayudaron claramente a facilitar que los alumnos entendieran el cambio de concepto, a nivel práctico todavía se observó grado de implicación y preparación muy mejorable.

No obstante, cabe añadir que las limitaciones que se siguen encontrando a este respecto son en su mayor parte intrínsecas a la asignatura, por el hecho de tratarse de una sesión única y tener un número muy elevado de alumnos (con grupos de prácticas heterogéneos, y frecuentemente numerosos a su vez), o a la naturaleza de las propias prácticas, en las que uno de los objetivos es el trabajo en equipo. Dado lo numeroso de los grupos, inevitablemente todo esto conlleva un reparto de tareas frecuentemente poco equitativo entre los distintos miembros de un grupo, de modo que se detectó que seguía habiendo un elevado número de alumnos que claramente confían en que no va a ser necesario ningún esfuerzo a nivel individual.

\subsubsection{Introducción de la evaluación competitiva: gamificación}

Con el fin de aumentar la preparación efectiva de la práctica, y viendo que la posibilidad de que se realizaran preguntas aleatorias sobre los contenidos, no había producido tampoco el efecto deseado, el tercer año se ha intentado fomentar la implicación de los alumnos 
mediante la introducción de un incentivo positivo. Para ello se ha recurrido al uso de la Gamificación Educativa como herramienta motivacional.

La gamificación es un recurso que cobra cada vez mayor importancia en las dinámicas motivacionales de las aulas (Marín Díaz, 2015). Según esta autora, la gamificación se puede definir como el empleo del juego como herramienta que facilita la cohesión del grupo y la motivación de los alumnos. Los resultados obtenidos con la gamificación son: la motivación, integración del grupo e impacto psicológico individual. Algunas experiencias previas han demostrado el potencial de esta herramienta para mejorar la adquisición de conocimientos. Rodríguez, Loro, \& Villén (2015) definen los cinco elementos que debe incluir una docencia basada en el juego:

a) Asignar un valor cuantitativo (la calificación)

b) Plantear un objetivo (la tarea)

c) Establecer formatos de clasificación (el nivel)

d) Ofrecer compensaciones (la recompensa)

e) Diseñar elementos de clasificación grupal (la integración)

Independientemente del perfil del alumno (los autores anteriores describen cuatro perfiles: competidor, conseguidor, socializador y explorador), la gamificación aprovecha la competitividad de los alumnos para conseguir una mayor motivación. Una de las aplicaciones de la gamificación como técnica educativa es la evaluación competitiva. Así, Iglesias-Rey \& Martínez-Solano (2017) emplean la evaluación competitiva como herramienta de evaluación de la docencia basada en proyectos obteniendo buenos resultados tanto a nivel de evaluación como de implicación del alumnado.

Siguiendo el ejemplo de los trabajos anteriores, en la documentación descrita en el apartado 3.1.1, se introdujo a propósito un error de concepto. El error aparecía en la parte III, donde se exponen los conceptos teóricos y procedimientos operativos, en concreto en el desarrollo y obtención de la expresión a utilizar para calcular, a partir de los datos experimentales obtenidos en el laboratorio, uno de los parámetros clave en uno de los tres ensayos (la $\mathrm{H}$ de la bomba), aplicando conceptos básicos de la mecánica de fluidos (ecuación de Bernouilli).

El día de la presentación de las prácticas, se les habló de la existencia de este error, y se planteó el incentivo. La evaluación se realizaría siguiendo dos criterios independientes. Por un lado se realizaría una evaluación clásica en función de los resultados presentados.Por otro lado, para cada grupo de teoría (5 en total como se ha mencionado en la introducción) el primer alumno de cada grupo que enviase un correo electrónico, identificando el error y justificando cuál sería la respuesta correcta, automáticamente obtendría una mejora en la nota de las prácticas de laboratorio. Una vez comentado en todos los grupos, se puso a su disposición en PoliformaT dicha documentación.

Para evitar que este incentivo no provocara una falta de atención en la práctica del alumno que lo consiguiera, y seguir manteniendo el interés del grupo en todo momento, se informó que no se confirmaría el resultado hasta una vez finalizadas todas las sesiones de prácticas.

(cc) EY-NC-ND 2018, Universitat Politècnica de València

Congreso IN-RED (2018) 


\section{Resultados}

El análisis de los resultados de la aplicación de la metodología basada en docencia inversa se realizará desde dos enfoques. Por un lado, se estudiará el impacto general sobre la asignatura en su conjunto a través del análisis de las encuestas que año tras año pasa el Instituto de Ciencias de la Educación (ICE) de la UPV a todos los alumnos matriculados. Por otra parte, como forma de incluir cierta retroalimentación a la aplicación de la metodología, durante el curso 2016-2017 se elaboraron las encuestas internas anteriormente mencionadas. En este curso 2017-2018 sin embargo, la iniciativa Proyecto Docencia Inversa de la UPV ha elaborado sus propias encuestas.

De las encuestas elaboradas por el ICE, cabe destacar el impacto positivo inicial en la aplicación de la metodología, tal y como se puede observar en la Figura 3.

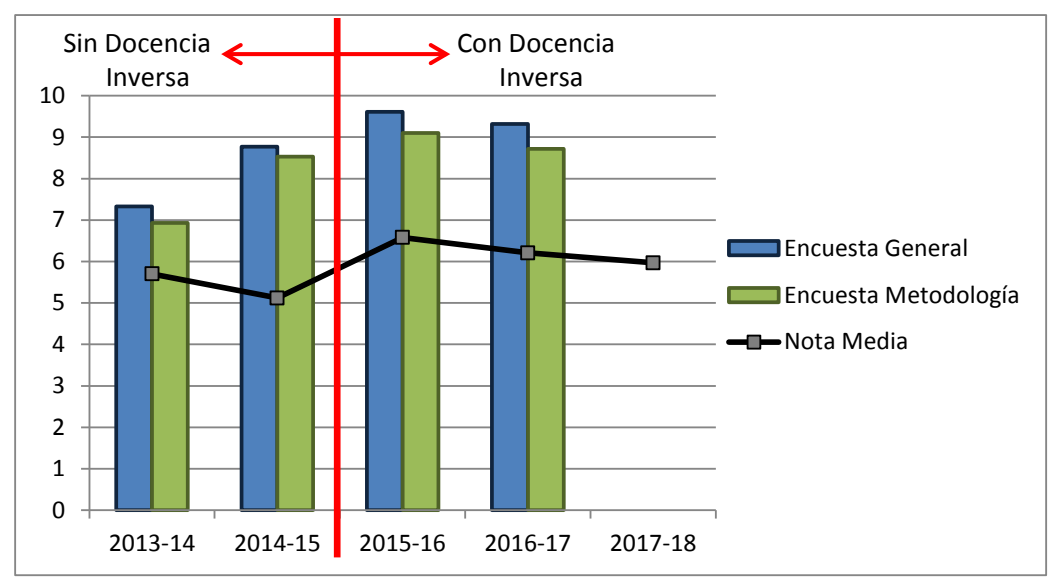

Figura 3. Evolución de la percepción por parte del alumno de la asignatura en general (barra azul), la metodología docente (barra verde) y de la calificación final de la asignatura. (Nota: las encuestas correspondientes al curso 2017-2018 no están disponibles en el momento de la elaboración de este trabajo)

Este análisis tiene la ventaja que afecta a la totalidad de los alumnos. Las encuestas tienen una larga tradición en la Universidad y llevan pasándose desde hace mucho tiempo. Por ello, el nivel de respuesta de los alumnos es elevado.

Las encuestas preparadas exprofeso tienen la ventaja de que están más dirigidas a evaluar la percepción de la metodología de docencia inversa. Sin embargo, al haber sido pasadas en el presente curso a través de la plataforma PoliformaT la respuesta recibida no ha sido tan amplia (se han recibido 14 encuestas de un total de 280 alumnos matriculados). Se constata en cualquier caso que de entre aquellos que sí han respondido a la encuesta, la percepción es positiva.

En este punto es necesario mencionar que el planteamiento de las encuestas específicas de docencia inversa en los dos cursos en los que se ha pasado es distinto. En el curso 20162017 la encuesta fue elaborada por los propios profesores y se intentó que fuese en la misma línea que las encuestas tradicionales, pero aplicada exclusivamente a las sesiones en las que la docencia inversa había sido aplicada. Así, las preguntas planteadas en la Figura 2 
se han sintetizado en las mismas categorías que las representadas en la Figura 3: Docencia General y Metodología. La Figura 4 recoge los resultados de esta encuesta interna.
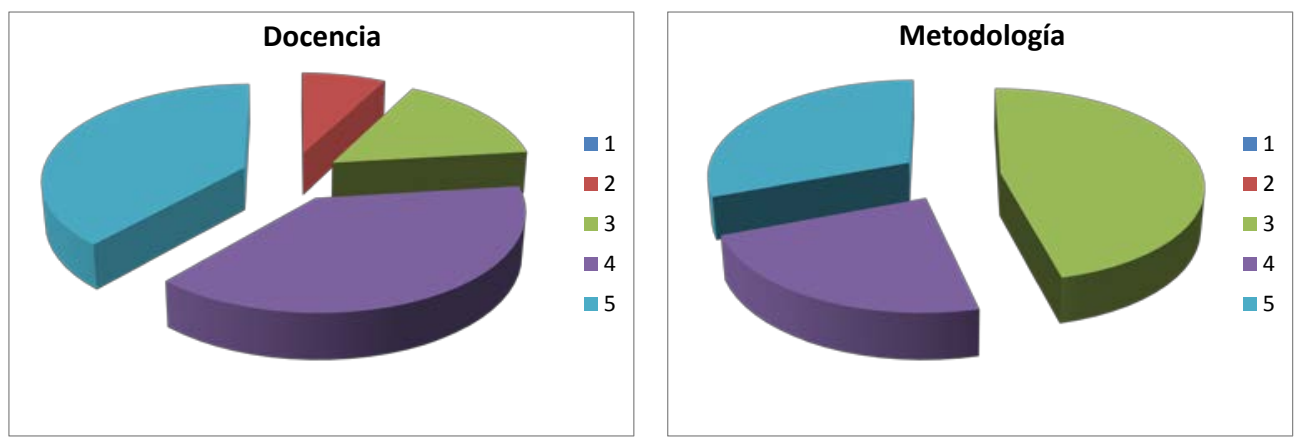

Figura 4. Resultados de la encuesta interna pasada sobre la percepción de la docencia inversa durante el curso 2016-2017. Izquierda: valoración de la docencia en general; Derecha: valoración de la metodología de Docencia Inversa. (1: Totalmente en Desacuerdo - 5: Totalmente de Acuerdo).

Por su parte, los resultados obtenidos por las encuestas específicas pasadas por el Proyecto Docencia Inversa son bastante similares. En este caso, las encuestas se han agrupado en tres grandes dimensiones:

a) Grado de satisfacción con la experiencia basada en la Docencia Inversa.

b) Recomendación de la metodología para otros compañeros que no la han seguido.

c) Propuesta de extensión de la metodología a otras asignaturas o campos dentro de la propia titulación.

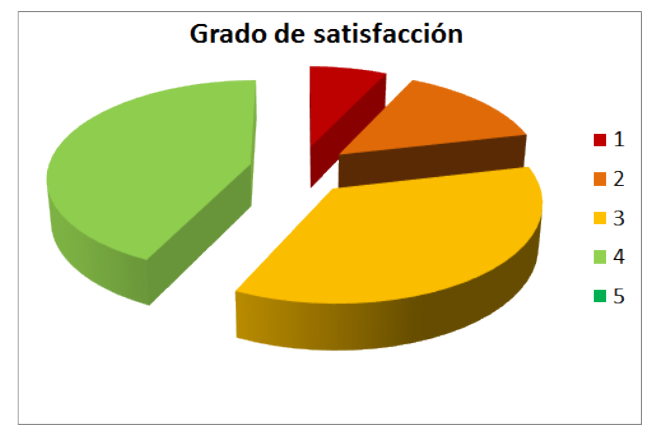

Figura 5. Encuesta del Proyecto Docencia Inversa. Grado de satisfacción.

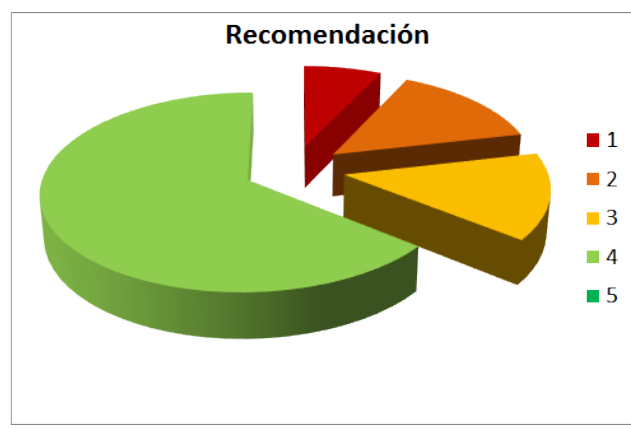

Figura 6. Encuesta del Proyecto Docencia Inversa. Recomendación a los compañeros.

(cc) EY-NC-ND 2018, Universitat Politècnica de València

Congreso IN-RED (2018) 


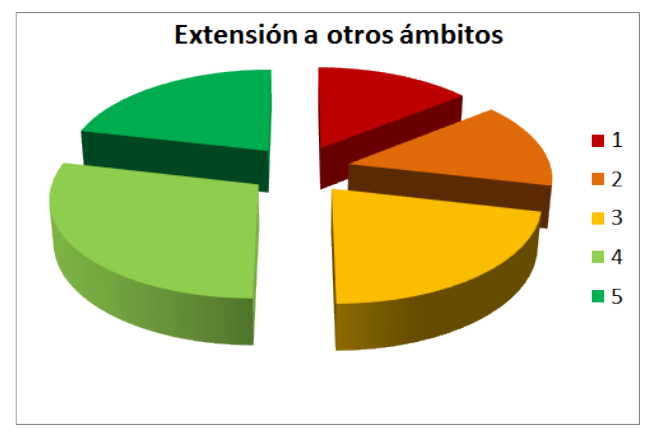

Figura 7. Encuesta del Proyecto Docencia Inversa. Extensión a otros ámbitos.

Los resultados de las encuestas clasificados en sus correspondientes dimensiones se muestran en las Figuras 5 a 7. La escala de respuestas varía desde 1 (Totalmente en Desacuerdo) hasta 5 (Totalmente de Acuerdo). Estos resultados se pueden clasificar como de aceptables, con una media global de la metodología de 3,26 sobre 5. Sin embargo, hay algunos aspectos que mejorar. En todas las dimensiones hay más opiniones favorables (4-5) que desfavorables (1-2). Esto es especialmente destacado en la recomendación a otros compañeros (2 de cada tres alumnos lo recomendaría). Sin embargo, una de las encuestas recogidas se ha mostrado muy crítica con la metodología, estando totalmente en desacuerdo con la misma. Además, se ha obtenido un nivel de respuestas bajo (un 5\%, 14 de 280). Esto hace que sea necesario plantearse los métodos de obtención de la retroalimentación.

\section{Conclusiones}

Se ha observado una evolución favorable a lo largo de estos tres años, en la implantación de la metodología e impacto en los resultados, considerando la experiencia como muy positiva en términos generales. Tras el balance a fecha actual se pueden apreciar de forma más clara las ventajas de la aplicación de esta metodología, en particular a prácticas de laboratorio, y con ello animar a docentes de asignaturas de esta magnitud (en cuanto a número de alumnos), en las que probablemente se observe una mayor complejidad o reticencia al cambio de metodología, a introducir este tipo de innovaciones de forma progresiva en sus materias. A la vista de los resultados de las encuestas (Figura 7), la mitad de los alumnos están de acuerdo o totalmente de acuerdo con esta afirmación.

Entre las ventajas se ha observado un progresivo aumento de la preparación de los alumnos a lo largo de los tres años que ha abarcado esta experiencia, que ha resultado evidente con el consecuente aumento en la participación y respuestas a las cuestiones que se plantean durante la breve introducción y revisión de los procedimientos realizada al inicio de la sesión. Además, la implantación de la metodología de Docencia Inversa muestra un efecto positivo tanto en la percepción de la asignatura por parte de los alumnos como en las calificaciones obtenidas (Figura 3).

Entre los cursos 2016-2017 y 2017-2018 (segundo y tercer año de implantación) se ha observado también una mayor calidad en la entrega de la parte IV (manipulación y análisis de resultados), especialmente en cuanto a conclusiones. Esto evidencia un gran aprovechamiento de la práctica, especialmente durante su desarrollo. 
Por otra parte, la aplicación de técnicas innovadoras en la actividad docente, y especialmente en el caso de la Docencia Inversa, requiere un trabajo continuo por parte del docente. Es por tanto importante tener presente que para poder aplicar la metodología tal y como aquí se ha descrito, es necesario realizar una revisión y actualización continua de la documentación cada curso para asegurarse que se mantiene totalmente la fidelidad a lo que el alumno va a encontrarse en el laboratorio y al procedimiento de ensayo que debe seguir.

La retroalimentación por parte del alumno continúa considerándose una herramienta fundamental para la mejora continua de resultados. En este sentido, claramente la posibilidad de realizar encuestas presenciales con un número no demasiado elevado de preguntas se considera puede aportar mucha mayor información. En cuanto a otro aspecto clave como es la toma de conciencia del alumno del cambio de metodología, resultaría bastante conveniente intentar aumentar las labores de concienciación por parte de la Universidad o de las Entidades Responsables de los Títulos (ERT) a nivel institucional. Esto podría realizarse de forma más evidente incluyendo la información sobre el tipo de metodología empleada incluso en el propio proceso de matrícula de las asignaturas.

De forma general, se considera que las principales limitaciones van más ligadas a la magnitud de la asignatura, y a la naturaleza de las clases (prácticas en grupo), que a la metodología en sí. Dado lo positivo de la experiencia, se plantea ampliar la metodología a otras partes de la asignatura en cursos posteriores.

\section{Agradecimientos}

La experiencia aquí descrita se ha llevado a cabo como parte de los trabajos desarrollados en el EICE DESMAHIA "Desarrollo de metodologías activas y estrategias de evaluación aplicadas al campo de la Ingeniería Hidráulica". Los autores agradecen a la UPV, y en particular al ICE, el apoyo prestado para llevar a cabo esta iniciativa.

\section{Referencias}

González Rogado, A. B., Vivar Quintana, A. M., Rodríguez Conde, M. J., Revilla Martín, I., Martínez Abad, F., Olmos Miguelañez, S., \& Barrientos Diego, P. (2014). Adquisición de la competencia de prevención de riesgos en el laboratorio mediante tecnología móvil. Congrés Internacional de Docència Universitària I Innovació (CIDUI), (1), 1-18.

Iglesias-Rey, P. L., \& Martínez-Solano, F. J. (2017). Combinación de una metodología basada en proyectos y de una evaluación competitiva en la asignatura Análisis y modelación de redes de distribución de agua. In Libro de Actas IN-RED 2017 - III Congreso Nacional de Innovación Educativa y de Docencia en Red. Valencia: Universitat Politècnica València. https://doi.org/10.4995/INRED2017.2017.6828

Llácer-Iglesias, R., \& Martínez-Solano, F. J. (2016). Experiencia en la aplicación de la docencia inversa para el aprendizaje de la asignatura de Máquinas Hidráulicas (Grado de Ingeniería en Tecnologías Industriales). In Libro de Actas IN-RED 2016 - II Congreso Nacional de Innovación Educativa y de Docencia en Red. Valencia: Universitat Politècnica València. https://doi.org/10.4995/INRED2016.2016.4288

Marín Díaz, V. (2015). La Gamificación educativa. Una alternativa para la enseñanza creativa. Digital Education Review, (27).

(cc) EY-NC-ND 2018, Universitat Politècnica de València

Congreso IN-RED (2018) 
Rodríguez, F., Loro, F., \& Villén, S. (2015). Experiencia de gamificación en alumnos de magisterio para la evaluación de la asignatura Sociología de la educación mediante el uso de la plataforma Kahoot. In INNODOCT 2015 (pp. 223-231). Valencia (España): Universitat Politècnica de València.

Sams, A., \& Bergmann, J. (2013). Flip Your Students’ Learning. Educational Leadership, 70(6), 1620.

(cc) EY-Nc-ND 2018, Universitat Politècnica de València 